\title{
Multidelayed random walks: Theory and application to the neolithic transition in Europe
}

\author{
Joaquim Fort, ${ }^{1}$ Debnarayan Jana, ${ }^{2,3}$ and Josep Humet ${ }^{4}$ \\ ${ }^{1}$ Departament de Física, Universitat de Girona, Campus de Montilivi, 17071 Girona, Catalonia, Spain \\ ${ }^{2}$ Center for Condensed Matter Sciences, National Taiwan University, 1 Roosevelt Road, Section 4, \\ Taipei, Taiwan 106, Republic of China \\ ${ }^{3}$ Department of Physics, University College of Science and Technology, 92 Acharya Prafulla Chandra Road, \\ Kolkata 700 009, WB, India \\ ${ }^{4}$ Departament d'Informàtica i Matemàtica Aplicada, Universitat de Girona, Campus de Montilivi, \\ 17071 Girona, Catalonia, Spain \\ (Received 11 March 2004; published 28 September 2004)
}

\begin{abstract}
We present a model in which particles (or individuals of a biological population) disperse with a rest time between consecutive motions (or migrations) which may take several possible values from a discrete set. Particles (or individuals) may also react (or reproduce). We derive a new equation for the effective rest time $\tilde{T}$ of the random walk. Application to the neolithic transition in Europe makes it possible to derive more realistic theoretical values for its wavefront speed than those following from the single-delayed framework presented previously [J. Fort and V. Méndez, Phys. Rev. Lett. 82, 867 (1999)]. The new results are consistent with the archaeological observations of this important historical process.
\end{abstract}

DOI: 10.1103/PhysRevE.70.031913

\section{INTRODUCTION}

The rest time of reproducing individuals causes a dispersive (as opposed to reproductive) delay in the spatial dynamics of the population density. The simplest description is that in which all individuals have the same rest time between any two consecutive jumps. In recent years, such single-delayed random-walk models have been successfully compared to observations of biophysical systems, including the speed of farming communities which gave rise to the neolithic [1,2], the speed of virus infections [3], etc. (for a review, see Ref. [4]). However, a single-delayed random walk is a highly idealized picture of the true microscopic motion of particles (or individuals, in biophysical applications). This is why several authors have gone beyond by considering several possible resting times for the particles (or individuals) in motion. Othmer, Dunbar, and Alt [5] considered a general framework. Later on, it was applied to compute the speed of reactiondiffusion fronts but, again, only for the single-delayed case [4]. Vlad and Ross [6] developed another model, and were the first to apply such an approach to a specific multidelayed example. They considered a gamma distribution of waiting times, which is realistic in many populations. But, as noted by Vlad and Ross [6] in their conclusions, data for preindustrial agriculturalists (see Sec. III below) are not detailed enough to believe that a gamma distribution is appropriate to the neolithic transition (and less still to fit a gamma distribution and estimate its parameter values with any confidence). Therefore, in this paper we would like to propose an alternative framework. We consider the case of several possible, discrete resting times, each of them with an associated probability, and determine the speed of wavefronts. We try to present a very clear, self-contained model from first principles (Sec. II), so that readers can understand the present paper without the need to resort to any additional source in the literature. Our assumption of several discrete resting times (each with a specific probability) is very useful because it makes it possible to apply data for preindustrial ag-
PACS number(s): 87.10.+e, 05.40.-a, 05.60.Cd, 47.70.-n

riculturalists in order to compute the predicted front speed of the neolithic transition in Europe (Sec. III), thus improving the framework of our previous, single-delayed model on the neolithic transition presented in Ref. [1]. Section IV is devoted to concluding remarks.

\section{THEORY}

In this section, we begin with the approach by Othmer, Dunbar, and Alt [5] to a system of particles with a distribution of delay times, but allowing also for the reproduction of particles or individuals [4], which leads to a variable total number of particles [7]. If $d s P(x, y, t)$ stands for the number of particles per unit area that reach an area $d s$ centered at $(x, y)$ at time $t$, we have $[5,4]$

$$
\begin{aligned}
P(x, y, t)= & \int_{0}^{t} d T \int_{-\infty}^{\infty} d \Delta x \int_{-\infty}^{\infty} d \Delta y P(x+\Delta x, y+\Delta y, t-T) \\
& \times \varphi(T) \phi(\Delta x, \Delta y)+\rho_{0} \delta(x=0) \delta(y=0) \delta(t=0) \\
& +F_{P}(x, y, t),
\end{aligned}
$$

where, following the same notation as in Ref. [1], $\phi(\Delta x, \Delta y)$ is the probability of making a jump of coordinate lengths $-\Delta x,-\Delta y$. Note that, in contrast to Ref. [1], we allow for several possible values of the rest time $T$ by using the probability $\varphi(T)$ that a particle rests for a time between $T$ and $T+d T$ before performing the next jump, divided by $d T$ [in Ref. [1] we assumed a Dirac delta, i.e., $\varphi(T)=\delta\left(T-T_{1}\right)$, i.e., the same rest time for all jumps]. We have used the product of probabilities $\varphi(T)$ and $\phi(\Delta x, \Delta y)$ in Eq. (1), and thus assumed that the rest times and lengths of jumps are uncorrelated (this is indeed justified, because such a correlation has not been observed in the phenomenon that we will analyze in Sec. III). The term $\rho_{0} \delta(x=0) \delta(y=0) \delta(t=0)$ corresponds to assuming that initially $(t=0)$ there are no particles at any point other than the origin [5], where the particle number 
density is $\rho_{0}$. The so-called source or growth function $F_{P}(x, y, t)$ is used to take care of the effect of the birth and death of individuals (or chemical reactions between particles), as usual [4].

The density $\rho(x, y, t)$ of particles (or individuals) per unit area centered at $\vec{x} \equiv(x, y)$ at time $t$ is clearly given by the particles that have arrived at $(x, y)$ at some earlier time and still not left, namely [5]

$$
\rho(x, y, t)=\int_{0}^{t} d t^{\prime} P\left(x, y, t^{\prime}\right) \Psi\left(t-t^{\prime}\right),
$$

where $\Psi\left(t-t^{\prime}\right)$ is the probability that any particle rests for at least a time interval $t-t^{\prime}$ before performing the next jump, obviously [5]

$$
\Psi\left(t-t^{\prime}\right)=\int_{t-t^{\prime}}^{\infty} d T \varphi(T)=1-\int_{0}^{t-t^{\prime}} d T \varphi(T) .
$$

In order to solve Eq. (1), we introduce the FourierLaplace transforms of the corresponding space-time fields $[8,9]$,

$$
\begin{aligned}
\hat{P}\left(k_{x}, k_{y}, s\right)= & \int_{-\infty}^{\infty} d x \int_{-\infty}^{\infty} d y \int_{0}^{\infty} d t e^{-i \vec{k} \cdot \vec{x}-s t} P(x, y, t), \\
\hat{\rho}\left(k_{x}, k_{y}, s\right)= & \int_{-\infty}^{\infty} d x \int_{-\infty}^{\infty} d y \int_{0}^{\infty} d t e^{-i \vec{k} \cdot \vec{x}-s t} \rho(x, y, t), \\
\hat{\varphi}(s) \hat{\phi}\left(k_{x}, k_{y}\right)= & \int_{0}^{\infty} d T e^{-s T} \varphi(T) \int_{-\infty}^{\infty} d \Delta x \int_{-\infty}^{\infty} d \Delta y \\
& \times e^{-i \vec{k} \cdot \overrightarrow{\Delta x}} \phi(\Delta x, \Delta y), \\
\hat{F}_{P}\left(k_{x}, k_{y}, s\right)= & \int_{-\infty}^{\infty} d x \int_{-\infty}^{\infty} d y \int_{0}^{\infty} d t e^{-i \vec{k} \cdot \vec{x}-s t} F_{P}(x, y, t) .
\end{aligned}
$$

Then, the Fourier-Laplace transforms of Eqs. (1) and (2) are [see, e.g., Ref. [8], formulas (F.5b,k) and (F.11g,n)]

$$
\begin{gathered}
\hat{P}\left(k_{x}, k_{y}, s\right)\left[1-\hat{\varphi}(s) \hat{\phi}\left(k_{x}, k_{y}\right)\right]=\rho_{0}+\hat{F}_{P}\left(k_{x}, k_{y}, s\right), \\
\hat{\rho}\left(k_{x}, k_{y}, s\right)=\hat{\Psi}(s) \hat{P}\left(k_{x}, k_{y}, s\right),
\end{gathered}
$$

where we have used the Laplace transform of Eq. (3), namely [see Ref. [9], formulas $(32.13,25)]$

$$
\hat{\Psi}(s)=\frac{1-\hat{\varphi}(s)}{s} .
$$

Therefore,

$$
\hat{\rho}\left(k_{x}, k_{y}, s\right)\left[1-\hat{\varphi}(s) \hat{\phi}\left(k_{x}, k_{y}\right)\right]=\frac{\rho_{0}-\hat{\varphi}(s)}{s}\left[1+\hat{F}_{P}\left(k_{x}, k_{y}, s\right)\right] .
$$

Antitransforming this equation, one can in principle find the differential equation for $\rho(x, y, t)$, which is the observable field in real space and time [10]. This framework was applied in Ref. [4] to the case of a single delay, namely $\varphi(T)=\delta(T$ $-T_{1}$ ) (which is, of course, nothing but a different route to the same result as that in Ref. [1]). In contrast, here we are interested in studying a system in which particles (or individuals) may wait for several possible rest times $\left(T_{1}, T_{2}, T_{3}, \ldots\right)$ before performing the next jump. Therefore, we will consider the distribution

$$
\varphi(T)=\sum_{i=1}^{N} p_{i} \delta\left(T-T_{i}\right),
$$

where $p_{i}$ is the probability that the rest time is $T_{i}$ (thus, $\sum_{i=1}^{N} p_{i}=1$ ). As explained in the Introduction (and explicitly shown in Sec. III), we consider a finite set of possible rest times $(i=1,2,3, \ldots, N)$ because in the application we are interested in, the available observations were recorded in this way, and fitting them to a continuous function (such as a gamma distribution) would introduce a very important source of error in the results, which would make them doubtful indeed. This difficulty of not having sufficiently detailed demographic dispersal data available has been already pointed out by previous authors [6].

Making use of Eq. (12) into Eq. (11),

$$
\hat{\rho}\left(k_{x}, k_{y}, s\right)\left[1-\left(\sum_{i=1}^{N} p_{i} e^{-s T_{i}}\right) \hat{\phi}\left(k_{x}, k_{y}\right)\right]=\frac{1-\sum_{i=1}^{N} p_{i} e^{-s T_{i}}}{s}\left[\rho_{0}+\hat{F}_{P}\left(k_{x}, k_{y}, s\right)\right] .
$$

In order to be able to antitransform this equation in the simplest possible way, we make the following steps.

First, we rewrite the previous equation in a form such that the time and space probability distributions do not appear as a product but as separate terms,

$$
\hat{\rho}\left(k_{x}, k_{y}, s\right)\left[\frac{1}{\sum_{i=1}^{N} p_{i} e^{-s T_{i}}}-\hat{\phi}\left(k_{x}, k_{y}\right)\right]=\frac{1}{s}\left[\frac{1}{\sum_{i=1}^{N} p_{i} e^{-s T_{i}}}-1\right]\left[\rho_{0}+\hat{F}_{P}\left(k_{x}, k_{y}, s\right)\right] .
$$


Second, as in Ref. [1], we assume that the space kernel is isotropic, i.e., $\phi(-\Delta x, \Delta y)=\phi(\Delta x, \Delta y)=\phi(\Delta x,-\Delta y)$ $=\phi(\Delta y, \Delta x)$. Then, from Eq. (6) and the normalization of probability $\left[\int_{-\infty}^{\infty} d \Delta x \int_{-\infty}^{\infty} d \Delta y \phi(\Delta x, \Delta y)=1\right]$, we find that

$$
\hat{\phi}\left(k_{x}, k_{y}\right)=\int_{-\infty}^{\infty} d \Delta x \int_{-\infty}^{\infty} d \Delta y\left[1-i \vec{k} \cdot \vec{\Delta}-k_{x}^{2} \frac{\Delta x^{2}}{2}-k_{y}^{2} \frac{\Delta y^{2}}{2}+O(3)\right] \phi(\Delta x, \Delta y)=1-\frac{\left\langle\Delta^{2}\right\rangle}{4}\left(k_{x}^{2}+k_{y}^{2}\right)+O(3),
$$

where $O(3)$ stands for terms of third and higher powers of $\Delta x$ and $\Delta y$.

Third, we assume that the characteristic time and space scales of the macroscopic observations (e.g., measurement of the front speed) are sufficiently large compared to the microscopic characteristic time and space scales (mean rest times $T_{i} \ll t$ and jump displacements $\Delta x \ll x, \Delta y \ll y$ ), so that we may approximate both sides of Eq. (14) by their Taylor series up to second order in the variables $T_{i}, \Delta x$, and $\Delta y$ [an equivalent assumption was made in Ref. [1], below Eq. (6)]. Then, Eq. (14) becomes

$$
\hat{\rho}\left(k_{x}, k_{y}, s\right)\left[\langle T\rangle s\left(1+\frac{\widetilde{T}}{2} s\right)+\widetilde{D}\langle T\rangle\left(k_{x}^{2}+k_{y}^{2}\right)\right]=\langle T\rangle\left(1+\frac{\widetilde{T}}{2} s\right)\left[\rho_{0}+\hat{F}_{P}\left(k_{x}, k_{y}, s\right)\right]
$$

where we have introduced the mean rest time between successive jumps as $\langle T\rangle=\sum_{i=1}^{N} p_{i} T_{i}$, the diffusion coefficient as

$$
\widetilde{D}=\frac{\left\langle\Delta^{2}\right\rangle}{4\langle T\rangle}
$$

which for a single rest time $\left(p_{1}=1, p_{2}=p_{3}=\cdots=0\right.$ [11]) reduces to the usual formula $D=\left\langle\Delta^{2}\right\rangle / 4 T_{1}[1,12]$, and we have defined

$$
\widetilde{T} \equiv 2\langle T\rangle-\frac{\left\langle T^{2}\right\rangle}{\langle T\rangle}=2 \sum_{i=1}^{N} p_{i} T_{i}-\frac{\sum_{i=1}^{N}\left(p_{i} T_{i}^{2}\right)}{\sum_{i=1}^{N} p_{i} T_{i}} .
$$

Equation (16) can be written

$$
\begin{aligned}
\frac{\widetilde{T} s}{2}\left(s \hat{\rho}-\rho_{0}\right)+s \hat{\rho}-\rho_{0}= & -\widetilde{D}\left(k_{x}^{2}+k_{y}^{2}\right) \hat{\rho}+\hat{F}_{P}\left(k_{x}, k_{y}, s\right) \\
& +\frac{\widetilde{T}}{2} s \hat{F}_{P}\left(k_{x}, k_{y}, s\right) .
\end{aligned}
$$

Antitransforming this equation, we obtain

$$
\frac{\widetilde{T}}{2} \frac{\partial^{2} \rho}{\partial t^{2}}+\frac{\partial \rho}{\partial t}=\tilde{D}\left(\frac{\partial^{2} \rho}{\partial x^{2}}+\frac{\partial^{2} \rho}{\partial y^{2}}\right)+F_{P}(x, y, t)+\frac{\widetilde{T}}{2} \frac{\partial F_{P}(x, y, t)}{\partial t},
$$

where we have used formulas (F.5j) in Ref. [8] and $(32.7,8)$ in Ref. [9], and applied that $\rho(x, y, t=0)=\rho_{0} \delta(x=0) \delta(y=0)$. We have also used that $F_{P}(x, y, t=0)=0$, which is justified if, as usual [4], we consider that the population is initially saturated at the origin and reproduces according to the logistic growth function, namely,

$$
F_{P}=a \rho\left(1-\frac{\rho}{\rho_{0}}\right),
$$

where $a$ is called the initial growth rate and $\rho_{0}$ the saturation density of the population. Equation (21) agrees with many experimental data on human populations $[13,14]$.

In our opinion, Eq. (20) is a very nice result. It shows that if there are several possible delays, the system still follows a hyperbolic reaction-diffusion equation [see Eq. (7) in Ref. [1]], but the role of the single rest time in the single-delay model in Ref. [1] is now played by $\widetilde{T}$, which is given by the new result (18). In this sense, $\widetilde{T}$ may be called an "effective" rest time. It may be written as

$$
\widetilde{T}=\langle T\rangle(1-\varepsilon),
$$

where

$$
\varepsilon=\frac{\left\langle(\Delta T)^{2}\right\rangle}{\langle T\rangle^{2}}=\frac{\left\langle(T-\langle T\rangle)^{2}\right\rangle}{\langle T\rangle^{2}}
$$

is the relative dispersion (or fluctuation) of the waiting time. Physically, we can understand the fact that $\widetilde{T} \neq\langle T\rangle$, i.e., the presence of the last term in Eq. (22), as follows. Consider two waiting-time distributions with the same mean $\langle T\rangle$ but different dispersions (Fig. 1). If the distribution shape is wide [Fig. 1(a)], some individuals will have low values of the dispersive delay $T$, as compared to those of narrower distribution [Fig. 1(b)]. Intuitively, it is obvious that a population front will travel faster if some individuals move sooner, i.e., with a lower delay $T$ [as in Fig. 1(a) as compared to Fig. 1(b)]. This is the physical reason why the effective delay $\widetilde{T}$ (which tends to slow the front down) will be lower for Fig. 1(a), so that $\tilde{T}$ decreases with increasing values of the dispersion $\varepsilon$, as predicted by Eq. (22).

It is easily seen (e.g., by considering the simple case of two possible delays $T_{1}$ and $T_{2}$ ) that it is possible that $\varepsilon>1$ and, therefore, $\tilde{T}<0$. In such an instance, Eq. (20) breaks down, as we will now show. In the derivation above of Eq. (16), we have Taylor-expanded the function [which appears in Eq. (14)] 


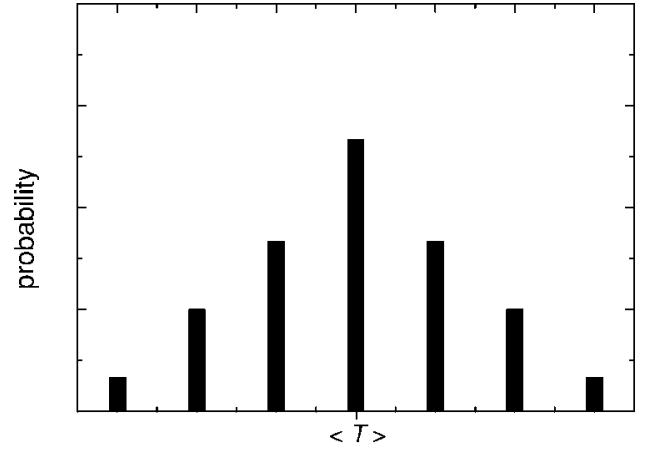

(a)

delay time $T$

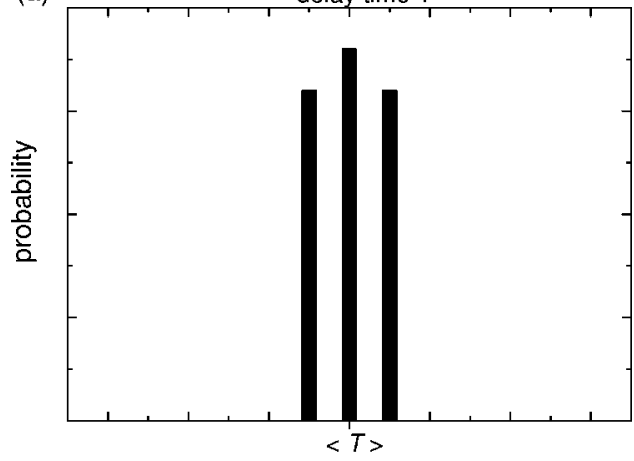

(b)

delay time $T$

FIG. 1. This figure is useful in understanding why the effective delay time $\tilde{T}$ of the random walk, introduced in this work, depends not only on the mean $\langle T\rangle$ but also on the dispersion $\varepsilon$ of the delaytime distribution. Both distributions depicted have the same mean delay $\langle T\rangle$, but that in (a) has a greater dispersion $\varepsilon$, implying that a few particles (or individuals) jump (or migrate) sooner (low values of $T$ ), which must lead to a faster front, i.e., to a lower effective delay $\widetilde{T}$. This explains that $\widetilde{T}$ decreases with increasing values of the dispersion $\varepsilon$, as predicted by Eq. (22).

$$
f \equiv \frac{1}{\sum_{i=1}^{N} p_{i} e^{-s T_{i}}}=1+\langle T\rangle_{s}+\frac{1}{2}\langle T\rangle \tilde{T} s^{2}+O\left(T_{i}^{3}\right)
$$

We note that $\partial f / \partial s>0$, and that for $\tilde{T}<0$ we have $\partial^{2} f / \partial s^{2}<0$. Thus, if $\tilde{T}<0$ the second-order approximation used in Eq. (16), namely $f \simeq 1+\langle T\rangle_{s}+\frac{1}{2}\langle T\rangle \widetilde{T} s^{2}$, becomes negative for some values of $s$, so that in this approximation we have that the function $\hat{\varphi}(s)=\sum_{i=1}^{N} p_{i} e^{-s T_{i}<0}$ for some values of $s$. But then Eq. (6) implies that the waiting-time probability distribution function $\varphi(T)<0$ for some values of $T$. Negative probabilities have no physical sense, thus we conclude that for $\tilde{T}<0$ the second-order expansion that leads to Eq. (20) cannot be applied. Of course, if in some specific application one computes the value of $\widetilde{T}$ using Eq. (18) and finds that $\widetilde{T}<0$, this does not mean that population fronts do not exist. One should then keep terms of order higher than the second in the Taylor expansions, so that Eq. (20) will be replaced by a more complicated equation with terms, e.g., of the form $\partial^{3} \rho / \partial t^{3}$. In the application we are interested in solving (Sec. III), this is not the case, i.e., we will find that $\tilde{T}>0$.
Therefore, in the present paper we will not analyze the case $\widetilde{T}<0$ in more detail. However, let us mention that we do not expect that analytical solutions are possible when additional terms in the Taylor series are kept, as already happens for the simpler, special case of a single delay even under some dispersal kernel assumptions that simplify the calculations [2].

Before leaving this section, we would like to stress the differences from previous work.

In Ref. [4], a single rest time between two successive jumps $\left[\varphi(T)=\delta\left(T-T_{1}\right)\right.$ [11]] and a Gaussian distribution of jump lengths in one dimension $\left[\phi(\Delta x) \propto \exp \left[-\Delta x^{2} / \alpha^{2}\right]\right]$ were assumed. In contrast, in the derivation above, (i) there are several possible, discrete values of the rest time [Eq. (12)]; (ii) the distribution of jump lengths is arbitrary; and (iii) space has two dimensions (as required for the application in the next section).

In Refs. $[6,16]$, the distribution of rest times applied is not given by Eq. (12) but by a gamma distribution (as explained in the Introduction, a gamma distribution is very useful for many cases, but not in the application that we analyze in the next section). Some other examples have also been considered in Ref. [17] (including non-Markovian random walks leading to anomalous diffusion). But instead of the very interesting, abstract, and general models in Refs. [6,17], for our purposes here it will be much simpler and clearer to make use of the direct approach we have presented. It makes the present paper self-contained and fits perfectly with the kind of data available for the problem we want to solve in the next section.

\section{APPLICATION TO THE NEOLITHIC TRANSITION IN EUROPE}

Reference [1] dealt with the range expansion of agriculturalists across Eurasia, which took place 10000 to 4000 years Before Present. In that model, a single rest time $T$ $=25 \mathrm{yr}$ was assumed and the evolution equation was

$$
\frac{T}{2} \frac{\partial^{2} \rho}{\partial t^{2}}+\frac{\partial \rho}{\partial t}=D\left(\frac{\partial^{2} \rho}{\partial x^{2}}+\frac{\partial^{2} \rho}{\partial y^{2}}\right)+F_{P}(x, y, t)+\frac{T}{2} \frac{\partial F_{P}(x, y, t)}{\partial t}
$$

with $F_{P}$ given by Eq. (21). The wavefront solutions to this equation have speed $[18,19]$

$$
v_{\text {single delay }}=\frac{2 \sqrt{a D}}{1+a \frac{T}{2}} .
$$

In Ref. [1], we also used the mean observed values $a$ $=0.032 \mathrm{yr}^{-1}$ and $\left\langle\Delta^{2}\right\rangle=1544 \mathrm{~km}^{2}$, as well as $D=\left\langle\Delta^{2}\right\rangle / 4 T$ in Eq. (25), and in this way obtained $v_{\text {single delay }}=1.0 \mathrm{~km} / \mathrm{yr}$, so that there was good agreement with the observed speed from the archaeological record $(1.0 \pm 0.2 \mathrm{~km} / \mathrm{yr}$, see Fig. 1 and the main text in Ref. [1], as well as [20]).

In [1], summarized in the previous paragraph, we simply assumed that the time between successive (i.e., parents' and their childrens') migrations is the same for all individuals, i.e., we made the approximation of a single rest time with a 


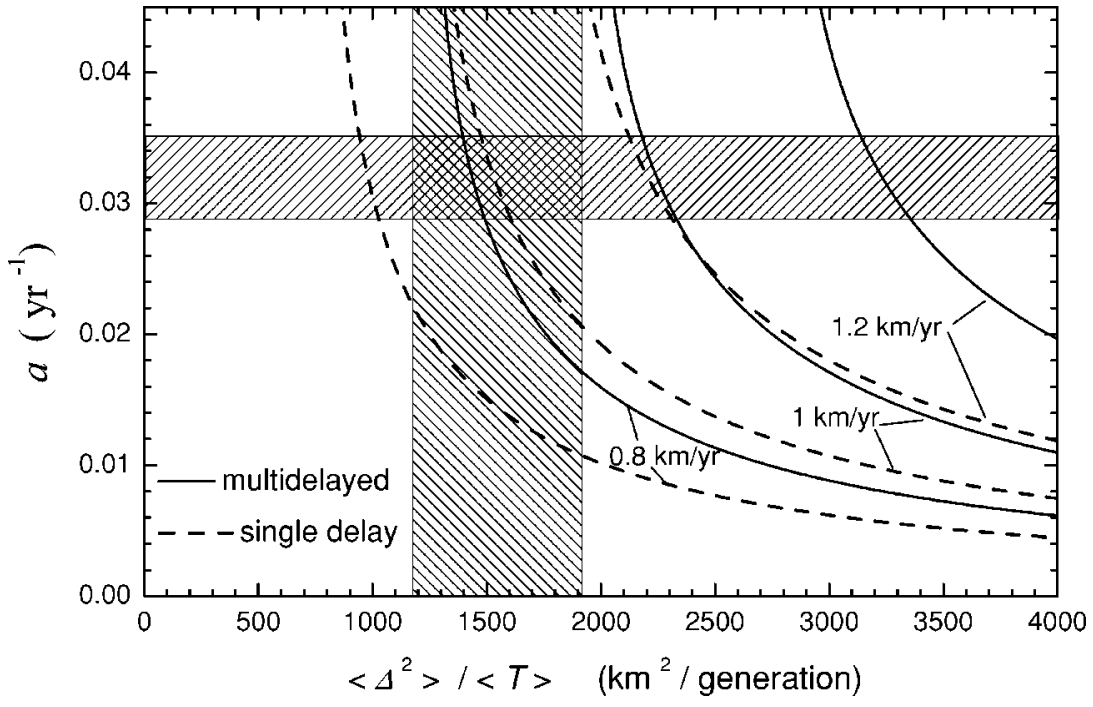

FIG. 2. Predictions for the speed of the wave of advance of farmers in the neolithic transition according to the model with a single delay [1] (dashed curves) and to the more realistic model with several discrete delays, as developed in the present paper (full curves). The dashed curves are therefore the same as those in Ref. [1], Fig. 3. It is seen that for many of the possible values of the reproductive and dispersive parameters of the population (hatched rectangle), the predictions of the new, more accurate model are still consistent with the front speed from the archaeological data $(1.0 \pm 0.2 \mathrm{~km} / \mathrm{yr}$, from Fig. 1 in Ref. [1]). value equal to the so-called generation time, namely $T$ $\simeq 25 \mathrm{yr}$ [15]. But, clearly, a single value for the rest time cannot be a realistic description, because different children will leave their parents at different ages. Therefore, it is quite important to see how the results change when taking into account a realistic rest-time distribution. In order to do so, we resort to Ref. [21], which is the same source from which the value of $\left\langle\Delta^{2}\right\rangle$ in Refs. [1,22] was estimated [23]. From Ref. [21], we find that the observed rest-time distribution is $T_{1}=27 \mathrm{yr}, p_{1}=0.46 ; T_{2}=35.5 \mathrm{yr}, p_{2}=0.51 ; T_{3}=45.5 \mathrm{yr}, p_{3}$ $=0.02 ; T_{4}=55.5 \mathrm{yr}, p_{4}=0.01$ [24]. Then Eq. (18) yields $\widetilde{T}$ $=31.1 \mathrm{yr}$. Using, for the moment, the same mean observed values as above, namely $a=0.032 \mathrm{yr}^{-1}$ and $\left\langle\Delta^{2}\right\rangle=1544 \mathrm{~km}^{2}$ [1], Eq. (17) yields $\tilde{D}=386 \mathrm{~km}^{2} / \mathrm{gen}=12.06 \mathrm{~km}^{2} / \mathrm{yr}$ and the wavefront speed of Eq. (20) is [18,19]

$$
v_{\text {multidelay }}=\frac{2 \sqrt{a \tilde{D}}}{1+a \frac{\widetilde{T}}{2}}=0.83 \mathrm{~km} / \mathrm{yr}
$$

which implies that the correction relative to the single-delay result in Ref. [1] is of $17 \%$, so that the effect of several delays turns out to be one of minor importance (as far as the order of magnitude is considered), but should not be neglected a priori. (Note that our detailed analysis of the demographic data is also relevant: the value $T=25 \mathrm{yr}$ used in Ref. [1] is the so-called generation time and was obtained from Ref. [22]. But we now realize that this is essentially the age difference between parents and their oldest child, not the mean age difference averaged over all children [24].) An important point is that the new, more accurate result of $0.83 \mathrm{~km} / \mathrm{yr}$ is still within the observed range for the wavefront speed $(1.0 \pm 0.2 \mathrm{~km} / \mathrm{yr}$, from Fig. 1 in Ref. [1]; see also [20]). Of course, our model is also interesting in itself, because it is applicable to many other human transitions and biological invasions, where the effect of having several delays may be a relevant point, as well as to nonbiological systems with random walks with several possible delays.
To what extent does our new result for the European neolithic depend on the uncertainties in the values of the parameters? In Fig. 2 we see that, for many values of $a$ and $\left\langle\Delta^{2}\right\rangle /\langle T\rangle$ in the range allowed by independent observations [1] (hatched rectangle), the predictions of the multidelayed model are consistent with the observed speed $(1.0 \pm 0.2 \mathrm{~km} / \mathrm{yr}[1])$.

\section{CONCLUDING REMARKS}

In this paper, we have presented a theoretical framework of multidelayed random walks with reaction. It has been applied to the neolithic transition in Europe. The effect of having several delays is, at the end, mathematically very simple: the single-delayed front speed is given by Eq. (25), whereas the multidelayed one is given by Eq. (26), which has the same mathematical form but with (i) the single rest time between successive jumps $T$ replaced by an effective rest time $\widetilde{T}$, which must be computed by means of our new Eq. (18), first derived in the present paper, and (ii) the diffusion coefficient $\widetilde{D}$ given by Eq. (17). In the specific application of the neolithic transition, the effect of a single delay was analyzed in Ref. [1]. Here, we have generalized it to the realistic case of several delays. The predicted speed is still consistent with the observed range of $1.0 \pm 0.2 \mathrm{~km} / \mathrm{yr}$ (Fig. 2), but it is somewhat lower, essentially because a reevaluation of the dispersal demographic data shows that the effective delay $\widetilde{T}$ is higher than the value $T=25 \mathrm{yr}$ used for the single-delay model in Ref. [1].

\section{ACKNOWLEDGMENTS}

We thank L. L. Cavalli-Sforza for suggesting this research work to us, for advice, and for discussions. Computing equipment used in this work was partially funded by the Generalitat de Catalunya under Grant No. Grup Consolidat SGR-2001-00186 (J.F.) and by the MICYT under Grant No. REN-2003-00185 (JF). 
[1] J. Fort and V. Méndez, Phys. Rev. Lett. 82, 867 (1999).

[2] J. Fort and V. Méndez, Phys. Rev. E 60, 5894 (1999).

[3] J. Fort and V. Méndez, Phys. Rev. Lett. 89, 178101 (2002).

[4] J. Fort and V. Méndez, Rep. Prog. Phys. 65, 895 (2002), especially Sec. 2.6.

[5] H. G. Othmer, S. R. Dunbar, and W. Alt, J. Math. Biol. 26, 263 (1998), especially pp. 268-270.

[6] M. O. Vlad and J. Ross, Phys. Rev. E 66, 061908 (2002).

[7] This causes the additional complication of normalizing probabilities, so in contrast to Refs. [5,4] we will deal with particle number densities per unit area instead of probabilities (also, in this way the comparison to the simpler, special case in Ref. [1] will be much clearer).

[8] J. D. Callen, Fundamentals of Plasma Physics (draft freely available on the Internet), Appendix B, Eqs. (F.5.b,k) and (F.11.g,n). Note that the variable $i \omega$ in this reference is equivalent to $-s$ in Ref. [9] and the present paper.

[9] M. R. Spiegel, Mathematical Handbook of Formulas and Tables (McGraw-Hill, New York, 1970), formulas 32.25 and 32.13.

[10] It is important to note that in this paper we have used the field $P(x, y, t)$ in addition to $\rho(x, y, t)$ because otherwise we cannot derive Eq. (11). In fact, we can derive an evolution equation for $\rho(x, y, t)$ [either from Eqs. (1) and (2) or intuitively], which turns out to be $\rho(x, y, t)=\int_{0}^{t} d T \int_{-\infty}^{\infty} d \Delta x \int_{-\infty}^{\infty} d \Delta y P(x+\Delta x, y$ $+\Delta y, t-T) \varphi(T) \phi(\Delta x, \Delta y)+\rho_{0} \delta(x=0) \delta(y=0) \int_{t}^{\infty} d T \varphi(t)$ $+\int_{0}^{t} d t^{\prime} F_{P}\left(x, y, t^{\prime}\right) \int_{t-t^{\prime}}^{\infty} d T \varphi(t)$, but we do not know how to derive the Fourier-Laplace transform of the last term. This is why we use $P(x, y, t)$ in addition to $\rho(x, y, t)$. Note also that the first term in the equation for $\rho(x, y, t)$ is the same as in Eq. (1) for $P(x, y, t)$, which is a way to realize that all equations in [1] hold for all values of $t$, not just for discrete ones, and that in [1] jumps can take place at any time (i.e., there is no need for jumps to take place all simultaneously at discrete values of $t$ ).

[11] It is worth noting that for the single-delayed case, namely, $\varphi(T)=\delta\left(T-T_{1}\right)$, Eq. (3) becomes $\Psi\left(t-t^{\prime}\right)=1$ if $t-t^{\prime}<T_{1}$ and $\Psi\left(t-t^{\prime}\right)=0$ if $t-t^{\prime}>T_{1}$ (as it obviously should), Eq. (2) reads $\rho(x, y, t)=0$ if $t<T_{1}$ (because all particles are still at the origin) and $\rho(x, y, t)=\int_{t-T_{1}}^{t} d t^{\prime} P\left(x, y, t^{\prime}\right)$ if $t>T_{1}$ (which means that all particles have arrived at most a time interval $T_{1}$ ago), and Eq. (1) becomes $P(x, y, t)=\int_{-\infty}^{\infty} d \Delta x \int_{-\infty}^{\infty} d \Delta y P(x+\Delta x, y+\Delta y, t$ $\left.-T_{1}\right) \phi(\Delta x, \Delta y)+\rho_{0} \delta(x=0) \delta(y=0) \delta(t=0)+F_{P}(x, y, t)$, where the first term means simply that the particles or individuals reaching $(x, y)$ at time $t$ after making a jump had possibly reached other points at time $t-T_{1}$. It is very important to mention that combining the last two equations and after a little algebra, one can recover Eqs. (1), (2), and (5) in Ref. [1] , as it should be.

[12] A. Okubo, Diffusion and Ecological Problems (Springer, Berlin, 1980).

[13] J. P. Birdsell, Cold Spring Harbor Symp. Quant. Biol. 22, 47 (1957).
[14] A. J. Lotka, Elements of Mathematical Biology (Dover, New York, 1956).

[15] Also, it is important to note that in Ref. [1] we allowed also for the possibility that some individuals do not move at all: it was included in the computation of the diffusion coefficient $D$ $=\left\langle\Delta^{2}\right\rangle /(4 T)$.

[16] S. Fedotov and Y. Okuda, Phys. Rev. E 66, 021113 (2002).

[17] S. Fedotov and V. Méndez, Phys. Rev. E 66, 030102 (2002).

[18] V. Méndez and J. Camacho, Phys. Rev. E 55, 6476 (1997).

[19] S. Fedotov, Phys. Rev. E 58, 5143 (1998).

[20] A new analysis of more recent archaeological data [M. Gkiasta, T. Russell, S. Shennan, and J. Steele, Antiquity 77, 45 (2003)] yields a similar but somewhat higher value for the speed, although its error is not estimated (in contrast, the data in Ref. [22] were used to compute both the time-versusdistance and the distance-versus-time speed errors in Ref. [1]). We plan to analyze the new data in detail in a separate publication.

[21] J. Stauder, The Majangir (Cambridge University Press, Cambridge, 1971).

[22] A. J. Ammerman and L. L. Cavalli-Sforza, The Neolithic Transition and the Genetics of Population in Europe (Princeton University Press, Princeton, 1984).

[23] Note that the value of $\left\langle\Delta^{2}\right\rangle$ is necessary to compute the diffusion coefficient, namely, $D=\left\langle\Delta^{2}\right\rangle /\left(4 T_{1}\right)$ for the single-delay model, as done in Refs. [1,22].

[24] Table 5 in Ref. [21], p. 58 gives the age distribution of unmarried people for preindustrial agriculturalists which (neglecting death effects, which we cannot estimate, as a first approximation) allows us to determine the number of people who have left a domestic group as $N_{1}=44, N_{2}=49, N_{3}=2$, and $N_{4}=1$ (from which our values of $p_{i}$ follow directly), with mean ages $a_{2}=27.5, a_{3}=37.5$, and $a_{4}=47.5$. Concerning $a_{1}$, in the same Ref. [21], p. 67, it is stated that children do not become marriageable (i.e., able to disperse according to the Majangir custom) until they are $a_{1} \simeq 15 \mathrm{yr}$ old. Note that these values of $p_{i}$ and $a_{i}$ yield a mean $\langle a\rangle \simeq 24 \mathrm{yr}$, which (adding about $1 \mathrm{yr}$ from the parents' migration until the child's birth) is indeed consistent with the value of $25 \mathrm{yr}$ for the generation time used in Ref. [1]. However, to apply the new model in the present paper, a final correction is necessary. If a son/daughter leaves their parents when he/she is, e.g., $19 \mathrm{yr}$ old, to this we should add the time interval from the migration of his/her parents until his/her birth. Since the mean number of children per family for preindustrial agriculturalists is about 6.5 [25] and their average birth interval is close to $2.5 \mathrm{yr}$ (Ref. [22], p. 66), we find that the mean time interval from the migration of the parents until a child's birth is of about $8 \mathrm{yr}$. Therefore, we have used $T_{i}=a_{i}$ $+8 \mathrm{yr}$ to obtain our values of $T_{i}$ in the main text.

[25] D. W. Sellen and R. Mace, Curr. Anthropol. 38, 878 (1997), table 2. 